\title{
¿Cómo incrementar el rendimiento diagnóstico del ultrasonido endoscópico?
}

\author{
Ángela Saúl-Pérez* \\ Departamento de Endoscopia Gastrointestinal, Instituto Nacional de Ciencias Médicas y Nutrición Salvador Zubirán, Ciudad de México, México
}

El ultrasonido endoscópico (USE) ha evolucionado en los últimos años posicionándose como un auxiliar terapéutico en diversas patologías gastrointestinales. Sin embargo, el aspecto diagnóstico del USE también ha evolucionado, ampliando aún más su utilidad y aplicación clínica. En el siguiente trabajo se describirán las contribuciones más destacadas presentadas durante la semana de enfermedades digestivas (DDW) 2021.

Desde el punto de vista de imagen, el USE con elastografía con ondas de cizalla o Shear Wave (SWE) se ha utilizado en la detección de cirrosis hepática al demostrar una correlación histológica ${ }^{1}$. Un modelo animal presentado como trabajo oral recomienda que la profundidad de la lesión sea $<2 \mathrm{~cm}$ y un tamaño entre 1-1.5 $\mathrm{cm}$ para optimizar resultados ${ }^{2}$.

Como se mencionó anteriormente este tipo de elastografía se utiliza principalmente en hígado con pocos estudios en patología pancreática. A este respecto durante la DDW 2021 se presentaron varios estudios que valoraron el papel de la SWE en páncreas. Uno de ellos demostró tener aplicación en la detección lesiones precursoras de cáncer de páncreas. Consistió en un estudio de 29 pacientes, el cual, a pesar de ser una muestra pequeña, abre la posibilidad de que el USE con SWE sea un marcador objetivo de lesiones precursoras tipo PanIN, al correlacionarlas con cambios similares a la pancreatitis crónica y adiposidad pancreática $^{3}$. De igual forma, un estudio prospectivo en 23 pacientes con diversas patologías pancreáticas, demostró que este tipo de elastografía es segura, con un éxito técnico del $100 \%$ y luego de 690 mediciones revela que la SWE parece tener más veracidad a nivel de la cabeza del páncreas ${ }^{4}$. Sin embargo, la SWE es una herramienta más de la cual hacen falta más estudios y que hasta el momento no puede sustituir a la elastografía de presión o strain elastography (ES). De hecho, se presentó un trabajo oral que valoró el potencial diagnóstico de la ES en cáncer de páncreas. Este estudio retrospectivo interesantemente concluye que la elastografía semicuantitativa puede reflejar características histológicas. Esto debido a que la elasticidad varía de acuerdo con el tipo histológico de cáncer y no se ve afectada por el tamaño del tumor. Incluso puede considerarse como factor pronóstico ya que el valor promedio de presión o MSV (por su nombre en inglés, mean strain value), correlaciona con la sobrevida. Este estudio tomó como punto de corte del MSV un valor de 30.5. Lesiones con $\geq 30.5$ se consideran lesiones blandas y este tipo de lesiones alcanzaron una sobrevida de $79 \%$ a 3 años comparada con una sobrevida de $33 \%$ en lesiones con valores por abajo del valor antes descrito ${ }^{5}$. Y asociado a la elastografía, un metaanálisis de 205 estudios reportó que la combinación de estas modalidades es confiable en la caracterización y diagnóstico de neoplasias pancreáticas al demostrar un valor predictivo positivo del $90 \% 6$.

No es de extrañar que nuevamente se presentaran trabajos relacionados con la adquisición de tejido. Uno de ellos fue un estudio aleatorizado controlado de 114 pacientes el cual no encontró diferencia en la agudeza diagnóstica entre la biopsia y la aspiración con aguja fina (FNB y FNA respectivamente). Encontrando la 
misma eficacia con dos pases asociados a citopatología in situ con cualquiera de estos dispositivos ${ }^{7}$. Esto contrasta con lo reportado en un estudio retrospectivo de 163 pacientes donde no se demostró una diferencia a favor de la citopatología rápida in situ (ROSE), ya que la agudeza diagnóstica de FNB con aguja 22 G fue > $96 \% \pm \mathrm{ROSE}^{8}$. Estos resultados son similares a otro trabajo presentado donde la agudeza diagnóstica de la FNA sin ROSE fue del $97 \%{ }^{9}$. Una de las modalidades en la toma de FNA es la FNA + USE harmónico, la cual mostró en un metaanálisis ser superior a la FNA convencional en el diagnóstico de lesiones pancreáticas (88.8 vs.83.6\%; $p<0.05$ ) sin diferencia en el número de pases ${ }^{10}$. Otro aspecto que nuevamente fue valorado fue el desempeño de la aguja biselada $20 \mathrm{G}$ la cual mostró una agudeza diagnóstica del $95 \%$ incluso en patólogos menos experimentados ${ }^{11}$. Y finalmente respecto a la adquisición de tejido, un campo creciente en la investigación actual es la biopsia hepática guiada por USE, la cual, de acuerdo con un estudio retrospectivo de 161 pacientes, mostró ser segura y eficaz con una correlación positiva entre la experiencia del operador y el número de espacios porta obtenidos ${ }^{12}$.

Respecto a las neoplasias quísticas de páncreas (NQP), un trabajo oral y un poster de una metaanálisis demostraron que bajos niveles de glucosa intraquística (corte $50 \mathrm{mg} / \mathrm{dl}$ ) tienen una sensibilidad del 90\% para diferenciar NQP mucinosas de las no mucinosas por lo que la medición de glucosa debe ser considerada como parte del abordaje estándar del análisis de líquido de las NQP ${ }^{13,14}$.

En relación con la detección del cáncer de páncreas, el enfoque actual es el diagnóstico temprano en pacientes de alto riesgo, para lo cual un estudio reportó que la adquisición de células neoplásicas circulantes en la vena porta mediante FNA es un método seguro y posible con mayor eficacia si se utiliza una aguja calibre $19 \mathrm{G}^{15}$. Mientras que otro estudio mostró que la medición de 5 biomarcadores en líquido pancreático y sérico tiene una especificidad del 100\% para cáncer de páncreas en población de alto riesgo ${ }^{16}$. $Y$ respecto al diagnóstico de cáncer en pacientes con estudios de imagen normales, una trabajo reportó que la dilatación del conducto pancreático principal y la raza caucásica, correlacionan con cáncer de páncreas en pancreatitis crónica. Mientras que la alteraciones parenquimatosas, la elevación de fosfatasa alcalina, bilirrubina y Ca 19-9 se asocian a cáncer en pancreatitis aguda idiopática ${ }^{17}$.

Por otro lado, sabemos que la pancreatitis autoinmune (PAl) comúnmente requiere de la confirmación histopatológica, sin embargo, la seguridad de la biopsia en este grupo de pacientes es debatible. Con base en esto, se presentó un estudio retrospectivo de 50 pacientes presentado de forma oral el cual encontró que el género masculino y una historia de otras enfermedades autoinmunes son factores a favor del diagnóstico de PAI. Y respecto al aspecto endosonográfico, concluye que un aspecto hipoecoico difuso del parénquima, un anillo anecoico y un engrosamiento de la pared del conducto biliar principal deben considerarse como hallazgos patognomónicos de PAI y que su presencia da más certeza para la toma de biopsia ${ }^{18}$.

Un área de gran interés es el impacto del USE en la medición del gradiente venoso portal (GVP), la cual es técnicamente posible y segura, correlaciona con parámetros clínicos de hipertensión portal al igual que con fibrosis demostrada histológicamente. Esto se mostró en dos trabajos orales, uno de los cuales demostró la seguridad de la medición del GVP por USE asociado a biopsia hepática guiada por USE ${ }^{19,20}$.

Finalmente, el desarrollo de organoides continúa creciendo. Dichos organoides se obtienen de células tumorales humanas cultivadas las cuales ayudan a comprender mejor la biología tumoral y guían la quimio-sensibilidad a diferentes fármacos. En este sentido un trabajo multicéntrico de 95 pacientes presentado de forma oral reportó que la adquisición de células para la elaboración de organoides puede llevarse a cabo de una biopsia guiada por USE y que el número de pases de la aguja, el retraso en el cultivo y la presencia de KRAS determinan el éxito de estos ${ }^{21}$.

Según los estudios antes mencionados, podemos concluir que las aplicaciones del USE continúan creciendo. Y citando una plática del estado del arte del USE, podemos decir que estas aplicaciones cambiarán el rol del endoscopista hacia un «oncólogo endoscopista», un «hepatólogo endoscopista» o un «radiólogo endoscopista».

\section{Financiamiento}

La presente investigación no ha recibido ayudas específicas provenientes de agencias del sector público, sector comercial o entidades sin ánimo de lucro.

\section{Conflicto de intereses}

Los autores declaran no tener conflicto de intereses. 


\section{Bibliografía}

1. Robles Medranda C, Oleas R, del Valle R, et al. Endoscopic ultrasound shear-wave elastography of the right and left hepatic lobe predicts liver cirrhosis: A diagnostic trial. Presentación oral, DDW 2021.

2. Wang T, Ryou M. ID: 3527010 Defining optimal technique for endoscopic ultrasound shear wave elastography (EUS-SWE): A combined benchtop and animal model study with comparison to transabdominal SWE. Gastrointest Endosc [Internet]. 2021;93(6 Suppl):AB229. Disponible en: https://www.giejournal.org/article/S0016-5107(21)00744-6/pdf

3. Chhoda A, Merenda S, Farrell J. ID: 3527197 Endoscopic ultrasound Shear Wave Elastography (SWE) for high risk individual pancreatic cancer surveillance. Gastrointest Endosc [Internet]. 2021:93(6 Suppl):AB262-AB263. Disponible en: https://www.giejournal.org/article/S0016-5107(21)00803-8/abstract

4. Kim K, Abboud Y, Samaan J, Park KH, Liu Q, Gupta K, et al. ID: 3526838 Endoscopic ultrasound guided Shear Wave Elastography (EUS-SWE) of the pancreas: Preliminary findings from a prospective registry. Gastrointest Endosc [Internet]. 2021;93(6 Suppl):AB237-AB238. Disponible en: https://www.giejournal.org/article/S0016-5107(21)00761-6/fulltext

5. Ohno $E$, Kawashima $H$, Ishikawa $T$, et al. Diagnostic potential of EUS-elastography as pathological or functional imaging for pancreatic cancer. Presentación oral, DDW 2021.

6. Shin C, Villa E. Sa1461 Contrast-enhanced endoscopic ultrasound combined with endoscopic ultrasound elastography for the diagnosis of pancreatic cancer. Gastrointest Endosc [Internet]. 2021;93(6 Suppl):AB200-AB201. Disponible en: https://www.giejournal.org/article/S0016-5107(20)31497-8/fulltext

7. Lubber DS, Boortalary T, Chiang AL, Coben RM, Kowalski TE, Loren DE. ID: 3524001 Randomized controlled trial comparing FNB needle to standard tip BNX in EUS-guided sampling of pancreas masses. Gastrointest Endosc [Internet]. 2021;93(6 Suppl):AB258. Disponible en: https://www. giejournal.org/article/S0016-5107(21)00796-3/abstract

8. Ansari Z, Varda B, Alani M, Pulivarthi V, Aslam S, Charley EL, et al. ID: 3526432 Diagnostic accuracy and procedure times of endoscopic ultrasound guided fine needle biopsy with and without rapid on-site evaluation using a 22 Gauge needle. Gastrointest Endosc [Internet]. 2021;93(6 Suppl):AB236. Disponible en: https://www.giejournal.org/article/S0016-5107(21)00758-6/fulltext

9. Alkhero M, Shalavadi M, Goraya A, Qureshi A, Tariq F, Chakrabarty I, et al. ID: 3516879 Clinical adequacy of endoscopic ultrasound-guided fine-needle aspiration in detecting malignancy without rapid onsite cytopathological evaluation: high demand surgical center experience. Gastrointest Endosc [Internet]. 2021:93(6 Suppl):AB219-AB220. Disponible en: https://www.giejournal.org/article/S0016-5107(21)00730-6/abstract

10. Ramai D, Facciorusso A, Mohan BP, Crinò SF, Ofosu A, Lisotti A, et al. ID: 3527230 Contrast-enhanced harmonic endoscopic ultrasound-guided fine-needle aspiration vs. standard fine-needle aspiration in pancreatic masses: A systematic review and meta-analysis. Gastrointest Endosc [Internet]. 2021;93(6 Suppl):AB253-AB254. Disponible en: https://www. giejournal.org/article/S0016-5107(21)00789-6/abstract

11. Omairi TW, Ardengh JC, Neto OM, et al. The diagnostic accuracy and tissue core acquisition of the novel forward-beveled 20-Gauge fine needle biopsy [Internet]. Digestive Disease Week® 2021, ePoster Library; 23 de mayo de 2021. Disponible en: https://eposters.ddw.org/ddw/2021/ddw-2021-virtual/320706/tarik.omairi.the.diagnostic.accuracy.and.tissue.core.acquisition. of.the.novel.html?f = menu\%3D6\%2Abrowseby\%3D8\%2Asortby\%3D6\%2Amedia\%3D2\%2Ace_id\%3D2020\%2Aot_id\%3D25386\%2Amarker\%3D1278
12. Bhurwal A, Gjeorgievski M, Delatore P, Tyberg A, Shahid HM, Abdelqader A, et al. ID: 3523451 Endoscopic ultrasound guided liver biopsy: A single center experience of bilobar liver sampling. Gastrointest Endosc [Internet]. 2021;93(6 Suppl):AB211-AB212. Disponible en: https://www.giejournal.org/article/S0016-5107(21)00714-8/abstract

13. Khan SR, Mohan BP, Madhu D, Chandan S, Kassab L, Ponnada S, et al. ID: 3520672 Intracystic glucose levels in differentiating mucinous from non-mucinous pancreatic cysts: A systematic review and meta-analysis. Gastrointest Endosc [Internet]. 2021;93(6 Suppl):AB263. Disponible en: https://www.giejournal.org/article/S00165107(21)00804-X/pdf

14. Satyavada S, Shaffer RS, Martínez B, et al. Diagnostic accuracy of intracystic concentrations of glucose and CEA in identifying mucin producing cysts neoplasm of the pancreas: A multicentric retrospective cohort study of histologically proven cysts. Presentación oral, DDW 2021.

15. Weston B, Coronel E, Lee JH, Guerrero PA, Hurd MW, Maitra A, et al. ID: 3526877 Endoscopic ultrasound-guided portal vein sampling if circulating tumor cells in pancreatic adenocarcinoma: A prospective randomized pilot study comparing a 22 and 19 Gauge needle. Gastrointest Endosc [Internet]. 2021;93(6 Suppl):AB230. Disponible en: https://www. giejournal.org/article/S0016-5107(21)00746-X/fulltext

16. Levink IJ, Visser IJ, Koopmann BD, et al. The diagnostic value of pancreatic juice protein biomarkers for pancreatic cancer detection. ePoster, DDW 2021.

17. Bartell N, Bittner K, Kothari T, Kaul V, Kothari S. ID: 3525792 Factors influencing the detection of pancreatic cancer not seen on cross-sectional imaging with endoscopic ultrasound in patients presenting with chronic or acute idiopathic pancreatitis. Gastrointest Endosc [Internet]. 2021;93(6 Suppl):AB255-AB256. Disponible en: https://www.giejournal. org/article/S0016-5107(21)00792-6/fulltext

18. Uberoi A, Yang JY, Taree A, Polydorides A, Dixon RE, Kumta NA, et al. ID: 3521665 Endoscopic ultrasound can identify pathognomonic features con autoimmune pancreatitis that distinguish from pancreatic cancer: A matched case-control study. Gastrointest Endosc [Internet]. 2021;93(6 Suppl):AB247. Disponible en: https://www.giejournal.org/article/S0016-5107(21)00776-8/abstract

19. Choi AY, Shah S, Kolb JM, Sowa P, Orozco D, Chahine A, et al. ID: 3522384 EUS-guided portal pressure gradient measurements predict fibrosis on liver histology. Gastrointest Endosc [Internet]. 2021:93(6 Suppl):AB231. Disponible en: https://www.giejournal.org/article/S00165107(21)00748-3/abstract

20. Kolb JM, Chang K, Wallace M, Bazarbashi AN, Cortés P, Brahmbhat B, et al. ID: 3522387 EUS-guided portal pressure gradient predicts clinical parameters of liver disease: The first multi-center experience of endohepatology. Gastrointest Endosc [Internet]. 2021;93(6 Suppl):AB231. Disponible en: https://www.giejournal.org/article/S0016-5107(21)00747-1/ abstract

21. Woo YJ, Nair KK, Shorr E, LaComb J, Kesar V, et al. ID: 3524866 EUSFNB derived human organoid models in pancreatic cancer: Clinical and technical predictors of success. Gastrointest Endosc [Internet]. 2021;93(6 Suppl):AB247-AB248. Disponible en: https://www.giejournal. org/article/S0016-5107(21)00777-X/abstract 